\title{
Mangelernährung im Mittelpunkt
}

\author{
Ein österreichischer Konsens gibt praxisnahe Empfehlungen
}

Mangelernährung ist eines der Gesundheitsprobleme alter Menschen in Pflegeheimen und Krankenhäusern: Auf geriatrischen Stationen etwa leidet mehr als die Hälfte der hochbetagten Senioren an Energie- und Nährstoffmangel. Dies verzögert ihre Genesung und verursacht weitere Probleme, von Infektionen bis zu Organschäden.

Ein gutes Ernährungsmanagement verbessert die Prognose dieser Patienten und verkürzt ihren Aufenthalt im Krankenhaus deutlich. Wie Mangelernährung durch systematisches Monitoring und Assessment in Pflegeeinrichtungen oder in der Pflege daheim möglichst früh erkannt werden kann und wie Ernährungs-Teams durch geeignete Maßnahmen gegensteuern können, stand im vergangenen Herbst in Wien bei der Präsentation des interdisziplinären Konsensus-Statements der Arbeitsgemeinschaft für Klinische Ernährung (AKE), der Österreichischen Gesellschaft für Geriatrie und Gerontologie (ÖGGG) und des Verbands der Diätologen Österreichs ebenso im Mittelpunkt wie bei einem Expertentreffen im Rahmen des Hauptstadtkongresses der Deutschen Gesellschaft für Anästhesiologie und Intensivmedizin (DGAI) in Berlin.

\section{Alarmierender Ernährungszustand in der Langzeitpflege}

„Mangelernährung bei alten Menschen zeigt nicht nur in Österreich, sondern in allen westlichen Industriestatten eine zunehmende Häufigkeit. Der Ernährungsstatus älterer Menschen in der Langzeitpflege ist alarmierend“, stellte Univ.-Prof. Dr. Erich Roth, Medizinisch Universität Wien und AKE beim „11. Österreichischen Kongresses für Führungskräfte in der Altenarbeit" Mitte September in Wien fest. Das KonsensusStatement „Geriatrie: Empfehlungen für die Ernährung des älteren Menschen in der Langzeitpflege" ist die Antwort auf die Ergebnisse des „NutritionDay in Pflegeheimen“. 17 Prozent der teilnehmenden Personen wiesen demnach Mangelernährung auf mit einem Body-Mass-Index (BMI) unter 20, bei 14 Prozent bestand ein Risiko für
Mangelernährung. Ungewollter Gewichtsverlust, ein Indikator für Mangelernährung, wurde bei neun Prozent verzeichnet. Die Nahrungsaufnahme der Mittagsmahlzeit war bei 33 Prozent unzureichend. „Ein schlechter Ernährungszustand bedeutet eine höhere Morbidität und eine schlechte Überlebensprognose für die folgenden sechs Monate“, so Roth: „Während die Sterblichkeitsrate bei den Heimbewohnern mit einem BMI über 22 bei zehn Prozent lag, erhöhte sie sich bei Bewohnern mit einem BMI zwischen 20 und 21,9 auf 17 Prozent und bei Bewohnern mit einem BMI unter 20

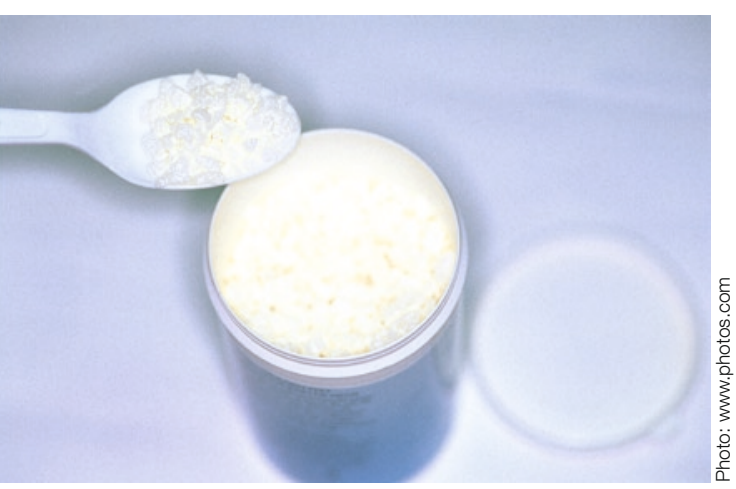

Schlechter Ernährungszustand bedeutet höhere Morbidität und chlechte Überlebensprognose für die folgenden sechs Monate.

auf 22 Prozent." Eine weitere Untersuchung des Ernährungszustand von 245 Pflegeheim-Bewohnern in Wien mit Pflegestufe 3 bis 5 mittels Mini Nutritional Assessment zeigte, dass nur 14 Prozent der Untersuchten einen zufriedenstellenden Ernährungsstatus hatten, 38 Prozent waren mangelernährt, bei 48 Prozent bestand ein Risiko dafür.

Hinter dem Problem können viele Ursachen stecken. „Gerade alte Menschen mit geistiger Beeinträchtigung achten oft wenig auf die Einnahme regelmäßiger Mahlzeiten“, erklärte Prof. Dr. med. Elisabeth Steinhagen-Thiessen, Leiterin des Evangelischen Geriatriezentrums Berlin (EGZB) beim Hauptstadtkongress der DGAI in Berlin. Aber auch Zahnprobleme oder Schluckstörungen können das Essen erschweren. Bei vielen Patienten behindern zudem etwa Darmerkrankungen die Aufnahme von Nährstoffen.

Praxisorientierte Empfehlungen für das systematische Erkennen von Malnutrition bei älteren Menschen in Pflege- und Seniorenheimen und in der Heimpflege sowie
Richtlinien, wie im Fall einer drohenden oder bereits bestehenden Mangelernährung vorzugehen ist, sollen die Ernährungssituation älterer Menschen verbessern.

\section{Routinemäßige Ernährungs- Screenings}

Eine Kernforderung des Papiers: „Alle Personen, die in eine Pflegeeinrichtung stationär aufgenommen werden, müssen bei der Aufnahme durch Pflegepersonen einem standardisierten Ernährungs-Screening unterzogen werden. Bei Bewohnern mit kognitiven oder kommunikativen Defiziten ist das betreuende Umfeld in die Erhebungsphase einzubinden." Erhoben werden Appetit- und Gewichtsverlust, akute Erkrankungen bzw. psychischer Stress während der vergangenen drei Monate ebenso wie der BMI, die Mobilität und die psychische Situation. Als Alternative zum zeitlich aufwändigeren Mini Nutritional Assessment, steht nun das Datenbankprogramm „NutriManager“ zur Verfügung. Damit werden Gewicht, gegessene Mahlzeiten und körperliche Aktivität der Patienten protokolliert. „Aus diesen Daten“, so Steinhagen-Thiessen „leiten wir dann den individuellen Energiebedarf ab und erstellen daraus einen Ernährungsplan."

Besteht der Verdacht eines Defizits, bedarf es zur weiteren Diagnose eines Assessments, das die Beeinträchtigungen, die zu Malnutrition führen können, spezifisch erfasst. Es bietet den Ansatz für aufzubauende pflegerische und therapeutische Maßnahmen mit dem Ziel des Sicherstellens einer ausreichenden Nahrungs- und Trinkmenge. Unterstützen kann hier zum Beispiel das Erstellen einer „Ess-Biographie“, die Vorlieben, Abneigungen und bevorzugte Essenszeiten erfasst, damit diese in der Praxis berücksichtigt werden können. Durch einen guten Ernährungszustand, so die Experten, können Krankenhausaufenthalte aufgrund von Folgeerscheinungen einer Mangelernährung reduziert werden.

Quelle: Presseaussendung zum 11. Österreichischen Kongresses für Führungskräfte in der Altenarbeit (15.-17.9. Wien); Presseaussendung HAI 2010 